\title{
Hat sich EMAS mit Erfolg überlebt?
}

\begin{abstract}
In Schleswig-Holstein hat die Vereinigung der Industrie- und Handelskammern 1999 mit dem Umweltministerium eine freiwillige Vereinbarung zu EMAS abgeschlossen, in der das Ziel gesetzt wurde, bis Ende 2003 die Zahl der EMASRegistrierungen auf 250 anzuheben. Inzwischen zeichnet sich jedoch ab, dass dieses Ziel nicht erreicht werden kann. Für die rückläufige EMAS-Beteiligung gibt es eine Reihe von Gründen.
\end{abstract}

$\mathrm{E}$ EMAS-Unternehmen in Schleswig-Holstein lässt sich nicht mehr revalidieren. In einer im Vorfeld eines zu diesem Thema veranstalteten Workshops durchgeführten Erhebung geben 7 der 14 befragten Unternehmen an, dass sie von EMAS auf ISO 14001 umsteigen bzw. die bisherige Doppelauditierung nach EMAS und ISO 14001 beenden werden (1). Die andere Hälfte der EMAS-Aussteiger will aber auch auf die ISO-Auditierung verzichten. Die Gesamtzahl der registrierten EMAS-Standorte nimmt nur deshalb nicht so stark ab, weil nach der Erweiterung des Anwendungsbereichs der Verordnung zunehmend Behörden, Verwaltungen und Dienstleister validierte Umweltmanagementsysteme einführen.

Diese Entwicklung ist nicht nur in Schleswig-Holstein zu beobachten. In einer derzeit vom Institut für ökologische Wirtschaftsforschung (IÖW) durchgefiuhrten Untersuchung zeichnen sich ähnliche Ergebnisse ab (2). Auch die bundesweiten Zahlen sprechen ein deutliche Sprache. Während nennenswerter Anteil der registrietten

die EMAS-Registrierungen in Deutschland über mehrere Jahre ihren Vorsprung gegenüber der ISO-Norm halten konnten, hat im Jahr 2002 nicht nur die ISO 14001 auch in Deutschland EMAS den Rang abgelaufen, sondern die Zahl der EMAS-registrierten Standorte bzw. Organisationen ist auch insgesamt rïckläufig (siehe Abbildung 1).

Zu befürchten ist nun, dass nach der zweiten EMASWelle der Behörden, Verwaltungen und Dienstleister die EMAS-Teilnahme mittelfristig deutlich zurückgehen könnte, und dass dies wiederum als ein Scheitern, sowohl von EMAS als auch grundsätzlich einer auf Freiwilligkeit setzenden Umweltpolitik, interpretiert würde. Dies könnte in der Konsequenz wieder zu einer stärkeren Orientierung auf Vorschriften führen. Deswegen gilt es die Ursachen für diese Entwicklung zu beleuchten, um die Erfolgsbeiträge der bisherigen Umweltpolitik benennen und Perspektiven aufzeigen zu können.

\section{Kosten-Nutzen-Verhältnis}

Die schleswig-holsteinische Umfrage macht deutlich, dass als zentrale Ursache für den Ausstieg

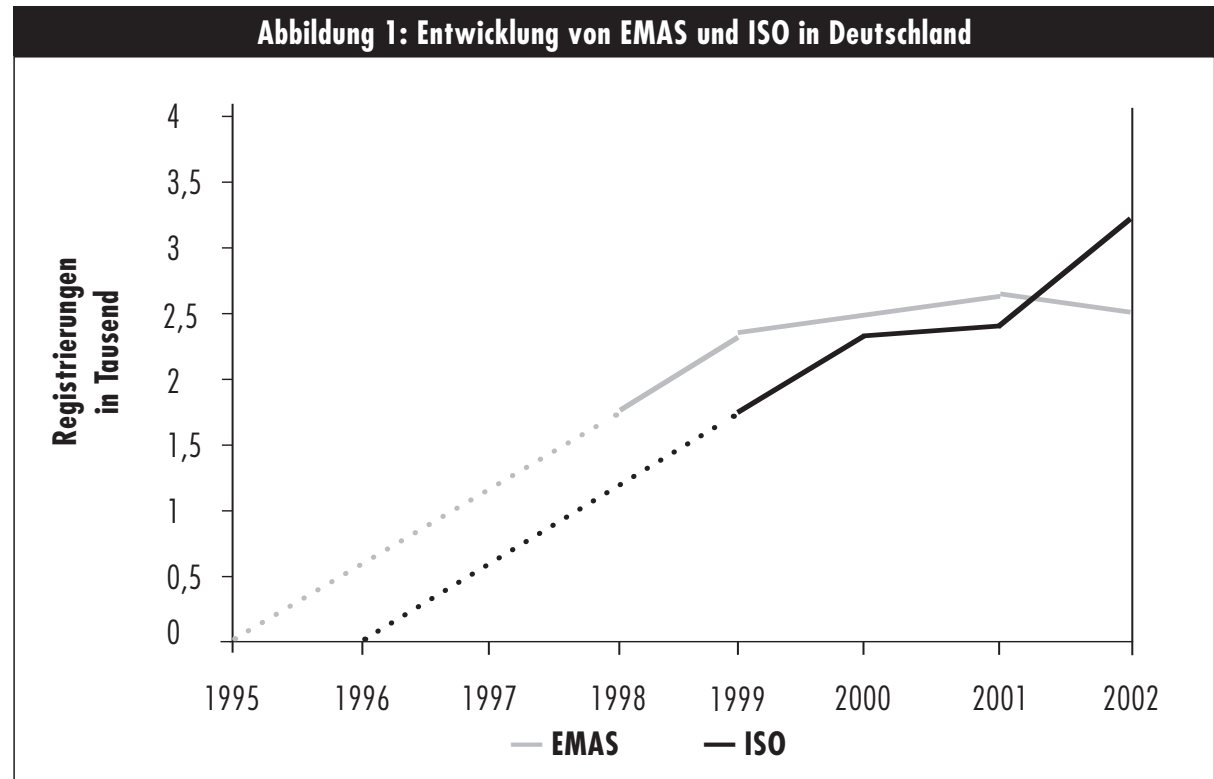

Quelle: eigene Darstellung nach Daten von Reinhard Peglau, Umweltbundesamt (3) aus EMAS das ungünstige Kosten-Nutzen-Verhältnis anzusehen ist. Hier wurden erste Warnsignale auch schon 1999 vom IÖW identifiziert (4). Viele Erwartungen, auf die bei der Einfuihrung des Umweltmanagementsystems gesetzt wurde, sind nicht eingetroffen. Große Enttäuschungen bestehen hinsichtlich der erhofften Marktvorteile und der Deregulierung. Andere Erwartungen sind zum Teil nur in deutlich geringerem Umfang eingetreten, wie zum Beispiel Kostensenkungen oder die Motivation der Mitarbeiter.

Weiterhin sind die Kosten für die Unternehmen bei EMAS höher als bei ISO 14001. Wesentlicher Faktor ist die Umwelterklärung, die erheblichen internen Aufwand sowie Layout- und Druckkosten verursacht, ohne dass damit aus Sicht der Unternehmen ein besonderer Nutzen verbunden ist. Es sind zwar Fälle bekannt, wo quasi aus der Not eine Tugend gemacht wurde, indem die Umwelterklärung als Imagebroschüre ausgebaut wurde, dennoch ist die Mehrheit der Unternehmen enttäuscht über das mangelnde Interesse bei ihren Kunden und der Nachbarschaft an dieser Berichterstattung. Auch Versuche über den Vertrieb die Umwelterklärung aktiv zu verbreiten, sind nicht zwingend erfolgreich. Weiterhin werden hohe Gutachterkosten und die zunehmend höheren Ansprüche der Gutachter bei der Auditierung beklagt. Die höheren Anforderungen von EMAS II schlagen sich in den Anforderungen der Audits nieder und verstärken somit die Problematik aus Sicht der auditierten Unternehmen.

Das bessere Kosten-Nutzen-Verhältnis der Zertifizierung nach ISO 14001 ist damit primär auf die niedrigeren Kosten und den niedrigeren Aufwand zurückzuführen. Die ursprüngliche Hoffnung seitens Politik und Umweltforschung, dass die Unternehmen bereit sind für EMAS als das bessere, weil anspruchsvollere System höhere Kosten und einen höheren Aufwand in Kauf zu nehmen, wird offensichtlich enttäuscht. Das „Sahnehäubchen“ wird auch den engagierten Unternehmen allmählich zu teuer.

Weiter sind die Fälle zu betrachten, denen auch ein extern auditiertes System nach ISO zu aufwändig ist. Hier handelt es sich insbesondere um kleinere Unternehmen aus weniger umweltrelevanten Branchen. Es ist davon auszugehen, dass sie unter Inanspruchnahme der KMU-orientierten Fördermittel EMAS eingeführt und erprobt haben und nun feststellen, dass diese aufwändige Form des Umweltmanagements nicht auf ihre Gegebenheiten passt. 


\section{- Nutzen unbestritten}

Trotz aller Kritik werden auch in SchleswigHolstein die Vorteile von EMAS und ISO 14001 geschätzt. Als Vorteile genannt werden die bereits in verschiedenen Studien festgestellten Nutzenkategorien, also gesteigerte Rechtssicherheit, verbesserte Organisation, verbesserte Einbindung der Mitarbeiter (nicht nur) in Umweltschutzfragen, Verbesserung der Umweltleistung und last but not least die Realisierung von Kostensenkungen. Allerdings muss man sich nach den bemerkenswerten Anfangserfolgen und Einsparungen auf Dauer inzwischen mit kleineren Verbesserungen zufrieden geben.

Dennoch bleibt die Notwendigkeit von Managementsystemen im Allgemeinen, ebenso wie die spezielle Bedeutung für den Umweltschutz bei größeren Unternehmen, unbestritten. Des Weiteren wird die regelmäßige externe Auditierung prinzipiell als Vorteil gesehen, da diese das Risiko des ,Einschlafens“ und der Stagnation des Systems mittelfristig verringert. Weitere Einflussfaktoren für die Durchführung eines Audits stellen die Branchenzugehörigkeit und die damit verbundene öffentlich wahrgenommene Umweltrelevanz dar. In einigen Branchen, zum Beispiel in der Automobilindustrie, wird von den Zulieferern ein auditiertes Umweltmanagementsystem auch in Zukunft vorausgesetzt. Je kleiner die Unternehmen sind, um so eher scheint das Kosten-NutzenVerhältnis aber problematisch.

\section{Deregulierung gewünscht}

Vielfach bemängelt wird von Seiten der Unternehmen, dass die erhofften Vorteile im Kontext von Berichtspflichten und sonstigen behördlichen Auflagen nicht eingetreten sind. Die Diskussion auf dem Workshop in Schleswig-Holstein machte deutlich, dass nicht zuletzt aufgrund der zunehmenden detaillierten Anforderungen aus Brüssel seitens der Behörden grundsätzlich wenig Spielräume für Deregulierungsmaßnahmen auf Länderebene gesehen werden. Wenn zum Beispiel Deutschland eine Berichtspflicht gegenüber der EU hinsichtlich bestimmter Emissionen hat, dann sind die Umweltbehörden gezwungen diese Informationen einzuholen.

In der Europäischen Kommission bestehen derzeit Überlegungen, ob Genehmigungspflichten für die nach EMAS validierten Unternehmen abgeschafft werden sollen. Unternehmensvertreter halten dem jedoch entgegen, dass die Genehmigung von Anlagen durchaus mit wichtigen Vorteilen verbunden ist, da so prinzipiell eine wesentlich höhere Rechts- sicherheit erreicht wird. Des Weiteren von Vorteil ist die Beratung der Behörden im Vorfeld, die im Kontext mit der Genehmigung genutzt werden kann. Liegen die Genehmigungen vor, dann können sie bei Bedarf auch im Umgang mit Nachbarschaftsbeschwerden verwendet werden. Schließlich ist bei der Auditierung das Vorliegen einer Genehmigung leichter zu prüfen als gegebenenfalls eine größere Vielzahl von Unterlagen. Damit sind im Bereich Deregulierung für EMAS-Unternehmen keine nennenswerten Vorteile zu erkennen oder in Zukunft zu erwarten.

\section{- Das Ende der Erfolgsstory}

EMAS ist eine deutsche Erfolgsstory, die sich nun überlebt. Die vor der ISO 14001 in Kraft getretene EMAS-Verordnung hat in zahlreichen Unternehmen zu einer Intensivierung und zu einer zunehmenden Systematisierung der Umweltschutzaktivitäten geführt. In den Anfangsjahren waren die Umwelterklärungen eher hilfreich als heute, obschon sie in der Regel nicht den ursprünglich erwarteten Zweck erfüllt haben. So trafen sie nicht auf das erwartete Interesse der Kunden und Nachbarn, dienten jedoch dem Erfahrungsaustausch zwischen den Unternehmen und der Umweltforschung. In einer Untersuchung des Zentrums für Europäische Wirtschaftsforschung in Kooperation mit dem IÖW gaben immerhin rund 20 Prozent der 1277 befragten Umweltbeauftragten an, dass sie aus Umwelterklärungen anderer Unternehmen Anregungen für ökologische Prozess- und Produktinnovationen gewinnen (5). Das Problem ist jedoch, dass aus einzelbetrieblicher Sicht den Kosten und dem Aufwand für die Erstellung einer Umwelterklärung nur ein geringer Nutzengewinn für das einzelne Unternehmen gegenüber steht.

Einen weiteren gravierenden Mangel sehen exportorientierte Unternehmen in der fehlenden internationalen Anerkennung der EMAS-Verordnung. Beide Gründe, also die höheren Kosten und die mangelnde internationale Anerkennung geben in zahlreichen Unternehmen den Ausschlag für den Wechsel von EMAS zu ISO 14001 oder sogar zu einem kompletten Ausstieg.

Prinzipiell wäre es daher dringend geboten,

- Fortschritte bei der Integration von EMAS in die europäischen und nationalen Regelwerke zu erreichen,

- EMAS endlich in der öffentlichen Beschaffung als Kriterium verwendbar zu machen und

- parallel dazu ein Kostensenkungsprogramm für EMAS zu entwickeln, sodass mittelfristig die
Kosten nicht mehr das Motiv für einen Wechsel zu ISO darstellen.

Leider dürften die zur Realisierung dieser Punkte nötigen Zeiträume durchweg deutlich länger ausfallen als die Bleibefrist vieler Unternehmen. Damit muss die Zukunft von Umweltmanagementsystemen in kleinen und mittleren Unternehmen als unsicher eingeschätzt werden. Große Unternehmen dagegen werden auch in Zukunft auf ISO 14 001 kaum verzichten.

Die Entwicklung der EMAS ist jedoch nicht als Scheitern, sondern als ein wichtiger Lernprozess anzusehen. Die EMAS-Unternehmen haben als Vorreiter wesentlich zur Weiterentwicklung von technischen und organisatorischen Umweltschutzinnovationen beigetragen. Und gerade heute lassen sich aus diesem Konsolidierungsprozess wichtige Rückschlüsse für die Weiterentwicklung von Umweltmanagementsystemen gewinnen.

\section{Anmerkungen}

(1) Der Workshop mit Umweltmanagern und Behördenvertretern, auf den sich dieser Beitrag zum Teil bezieht, fand im №vember 2002 in Kiel statt. Vgl. Ministerium für Umwelt, Natur und Forsten des Landes Schleswig-Holstein, IHK Vereinigung Schleswig-Holstein (Hrsg.): Zukunft von EMAS in Schleswig Holstein. Dokumentation des Fachworkshops, Kiel 2002.

(2) Monitoring von Umweltmanagement und Umweltmanagementsystem. Letzte Durchführung der sechsjährigen $\mathrm{Pa}$ nelbefragung. Im Auftrag der Hessischen Landesanstalt für Umwelt und Geologie. Das Vorhaben wird im März 2003 abgeschlossen.

(3) Die so genannten Peglau-Listen werden von Reinhard Peglau (Umweltbundesamt) in regelmäßigen Abständen erstellt und im Internet angeboten (www. 14001 news.de). Während die Zahlen zu EMAS von Registrierungsstellen systematisch erfasst werden, beruhen die Zahlen zu ISO auf Umfragen bei Experten und sind daher mit einer gewissen Unsicherheit behaftet.

(4) Vgl. Hessisches Landesamt für Umwelt und Geologie (Hrsg.): Monitoring von Umweltleistung und Umweltmanagementsystem, Befragung 1999, Wiesbaden 2000, S. $19 f f$.

(5) Vgl. Rennings, K./ Ankele K./ Hoffmann, E./ Nill, J./ Ziegler, A.: Innovationswirkungen des EG-Umwelt-Auditsystems in Deutschland - Eine Analyse auf Basis von Fallstudien und einer Breitenbefragung. Mannheim/ Berlin, Januar 2003 (noch unveröffentlichter Abschlussbericht).

\section{Der Autor}

Thomas Loew ist wissenschaftlicher Mitarbeiter im Forschungsfeld Ökologische Unternehmenspolitik des Instituts für ökologische Wirtschaftsforschung (IÖW). Kontakt: IÖW, Potsdamer Str. 105, 10785 Berlin. Tel. 030-884594-17, Fax 8825439,

E-Mail: Thomas.Loew@ioew.de 
(c) 20I0 Authors; licensee IÖW and oekom verlag. This is an article distributed under the terms of the Creative Commons Attribution Non-Commercial No Derivates License (http://creativecommons.org/licenses/by-nc-nd/3.o/), which permits unrestricted use, distribution, and reproduction in any medium, provided the original work is properly cited. 